ISSN 1409-2441
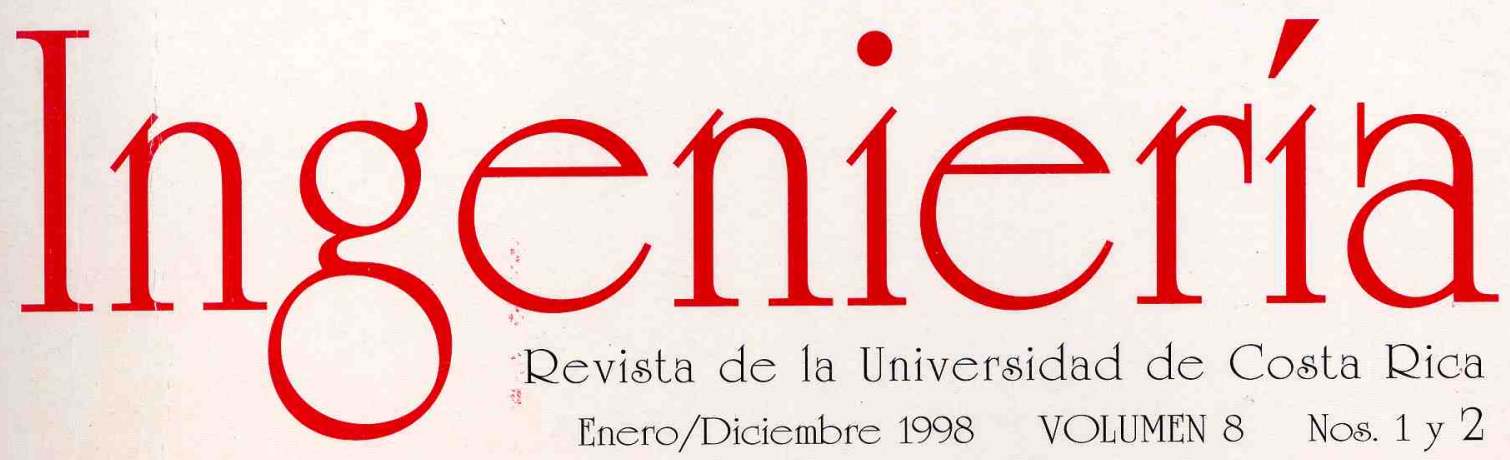

Revista de la Universidad de Costa Rica Enero/Diciembre 1998 VOLUMEN 8 Nos. 1 y 2 


\section{PROYECTOS DE INVESTIGACION y ACCION SOCIAL DE LA FACULTAD DE INGENIERIA VIGENTES A 1998}

\author{
ESCUELA \\ DE \\ INGENIERIA \\ A GRIC OLA

\section{Investigación}

Definición de planicies de inundación usando modelos hidrológicos, hidráulicos y de sistemas de información geográfica.

No. 340-97-334. Hernán Solís Bolaños

Metodología de enlace de un sistema de simulación agrícola y/o ambiental y un sistema de información geográfica.

No.340-97-335. Francisco Aguilar Pereira; Hernán Solís Bolaños.

\section{Acción Social}

Identificación de micro-proyectos de riego para su financiamiento.

No. 0428010. José Rafael Rojas Bolaños.

Mejor Manejo de Fertilizantes en Suelos Tropicales: Foro de Extensión.

No. ED-12-97. Ricardo Radulovich Ramírez.

\section{ESCUELA CIENCIAS DE LA COMPUTACIÓN E INFORMÁTICA}

\section{Investigación}

Programa de investigación en bases de datos.

No. 362-97-202. Dr. Ronald Arguello V., M.Sc. Elka Malinowski G. y M.Sc. Javier Gainza E.

Extracción inductiva de reglas de asociación de grandes bases de datos.

No. 326-97-316. Dr. Ronald Arguello V. y M.Sc. Elka Malinowski G.

Algunos factores que afectan la escogencia de la carrera de cómputo de la UC.R.

No. 326-98-363. M.Sc. Silvia Chavarría G.
Polimorfismo Uniforme más eficiente. No. 326-98-391. M.Sc. Adolfo Di Mare H.

Banco de Germoplasma del Pejibaye.

No. 111-79-908. M.Sc. Javier Gainza E.

Sistemas de información e Ingeniería de Software.

No. 326-98-901. Dr. Marcelo Jenkins C., Dra. Gabriela Marín R. y Dr. Ronald Arguello V.

Diseño e implementación de planes de aseguramiento de la calidad para el desarrollo del software.

No. 326-98-240. Dr. Marcelo Jenkins C., Dra. Gabriela Marín R. y Dr. Ronald Arguello V.

Desarrollo y utilización de la realidad virtual.

No. 326-97-314. M.Sc. Carlos Vargas C.

Sistemas de aprendizaje híbridos (SAH): potencial y factibilidad de aplicación en Costa Rica.

No. 326-99-302. Dra. Yadira Solano S.

\section{Acción Social}

Proyección a sectores de Escasos Recursos, TCU

No. 0427060. Licda. Roxana Vargas S.

Apropiándonos de la informática, TCU

No. 0427020. Lic. Alan Calderón C.

Programa de asesoría, actualización y capacitación computacional.

No. 211. M.Sc. Silvia Chavarría G. 
ESCUELA DE INGENIERIA QUIMICA

\section{Investigación}

Establecimiento de un paquete tecnólogico para producción de moldes metálicos en la industria plástica.

No. 325-94-234. Dr. Manuel Calvo F. y Dr. Gerardo Rojas Meza.

Investigación en relación a la purificación del baño metálico de aleaciones de estaño y plomo, para mejorar propiedades estructurales.

No. 325-97-524. Vigencia del 01-01-99 al 31-12-99. Dr. Manuel Calvo F.

Evaluación de la Resistencia a la Durabilidad de 10 especies maderables de Costa Rica.

No. 731-95-251. Vigencia del 01-01-99 al 31-12-99. M.Sc. Lorena Blanco R.

Propiedades y uso de las maderas del Valle Central de Costa Rica

No. 731-96-295. Vigencia del 01-01-99 al 31-12-99. M.Sc. Lorena Blanco R.

\section{Acción Social}

Asesoría a la Pequeña Industria. No. 425010. M. Sc. Hernán Camacho Soto
Protección Integral Industrial: Salud e Higiene Ocupacional, Prevención y Control Ambiental

No. 425030. Dr. Harry Castillo Valle.

\section{ESCUELA DE INGENIERIA ELECTRICA \\ Investigación}

Seminario - taller de métodos y técnicas de investigación y de redacción de trabajos científicos.

No. 4292-1. Dr. Wilbert Ezequiel Solano Rojas.

Tutoría en métodos de elaboración y redacción de trabajos científicos.

No. 4292-2. Dr. Wilbert Ezequiel Solano Rojas.

Curso de técnicas para una comunicación eficaz.

No. 4292-3. Dr. Wilbert Ezequiel Solano Rojas.

Consultoría y asesoría técnica en planificación de sistemas de formación de recursos humanos.

No. 4391-00 ó 6291-00. Dr. Wilbert Ezequiel Solano Rojas.

\section{Acción Social}

Cursos de Capacitación permanente.

No. 12995.00. Ing. Luis Diego Marín. 